ISSN 0103-5150

Fisioter. Mov., Curitiba, v. 25, n. 3, p. 517-524, jul./set. 2012

Licenciado sob uma Licença Creative Commons

\title{
Relação entre os desempenhos em diferentes testes frequentemente utilizados na avaliação da função manual
}

\author{
Relationship among performance in different tests \\ often used for hand function assessment
}

\section{Kauê Carvalho de Almeida Lima ${ }^{[a]}$, Matheus Menezes Francisco ${ }^{[b]}$, Paulo Barbosa de Freitas ${ }^{[c]}$}

[a] Fisioterapeuta e mestrando do Programa de Pós-Graduação em Ciências do Movimento Humano, Universidade Cruzeiro do Sul, São Paulo, SP - Brasil, e-mail: kaue.alima@gmail.com

[b] Graduando de Educação Física e aluno de iniciação científica pela Universidade Cruzeiro do Sul, São Paulo, SP - Brasil, e-mail: matheus.f.menezes@gmail.com

[c] Doutor em Biomecânica e Ciências do Movimento, professor adjunto da Universidade Cruzeiro do Sul, São Paulo, SP Brasil, e-mail: defreitaspb@gmail.com

\section{Resumo}

Introdução: Testes de Força de Preensão Palmar Máxima $\left(\mathrm{FP}_{\mathrm{Max}}\right)$ e de destreza manual são utilizados como indicativos da função manual . Porém, a $\mathrm{FP}_{\mathrm{Max}}$ pode ter sua validade questionada, em virtude de raramente exercermos força máxima em tarefas cotidianas. Para investigar a validade da $\mathrm{FP}_{\mathrm{Max}}$, examinamos a relação dessa variável com outras obtidas em testes de destreza manual. Ainda, investigamos a relação entre testes de destreza manual. Objetivo: Verificar a relação entre a $\mathrm{FP}_{\mathrm{Max}}$ e o desempenho no Teste de Função Manual de Jebsen e Taylor (TFMJT) e entre o desempenho nesse teste e o desempenho no teste dos Nove Pinos nos Buracos (9-PnB). Método: Trinta e seis adultos jovens e sadios (18 homens e 18 mulheres) realizaram o TFMJT, seguido do 9-PnB e do teste de $\mathrm{FP}_{\mathrm{Max}}$, com ambas as mãos. Foram realizadas análises de correlação entre o desempenho nesses testes. Resultados: Os resultados revelaram a existência de uma relação negativa moderada entre a $\mathrm{FP}_{\mathrm{Max}}$ e o TFMJT $(\mathrm{r}<-0,74)$, mostrando que, quanto mais forte o indivíduo, menor é o tempo para realizar o TFMJT. Os resultados revelaram, também, uma fraca correlação entre o teste 9-PnB e o TFMJT, somente na mão não dominante. Conclusões: A FP $_{\text {Max }}$ pode ser utilizada como indicativo da FM, mas 
seus resultados devem ser utilizados com cautela, pois essa variável não contempla todos os aspectos que envolvem a coordenação e o controle das forças atuantes durante a manipulação de objetos. Os testes 9-PnB e TFMJT indicam características diferentes relacionadas às capacidades manipulativas.

Palavras-chave: Função manual. Destreza. Dinamômetro de força muscular.

\begin{abstract}
Introduction: Tests that measure the maximum grip strength ( $\left.G S_{\text {Max }}\right)$ and hand dexterity as the Nine Hole Peg Test (9-HPT) and the Jebsen \& Taylor Hand Function Test (JTHFT) are commonly used to assess hand function (HF). However, $G S_{\text {Max }}$ may be questioned regarding its validity because we rarely exert maximum grip force to perform everyday manipulation tasks. To explore $G S_{\text {Max }}$ validity we investigated the relation between $G S_{\text {Max }}$ and tests that assess hand dexterity. Also, we tested the relationship between tests used to evaluate hand dexterity. Aim: To assess the relationship between $G S_{\text {Max }}$ and the performance in JTHFT and the relationship between the performance in 9-HPT and JTHFT. Method: Thirty-six healthy young adults (18 males and 18 females) performed the JTHFT followed by the 9-HPT and the $G S_{\text {Max }}$ test, with their dominant and non-dominant hands. Pearson's correlation analyses were performed between them. Results: Moderate negative correlation between the $G S_{\text {Max }}$ and the JTHFT was revealed ( $\left.r<-0.74\right)$, indicating that stronger individuals perform the JTHFT faster. Also, results revealed a weak correlation between the performance in 9-HPT and the JTHFT, and only for the non-dominant hand. Conclusion: $G S_{\text {Max }}$ can be used as an indicator of HF, but it should be used prudently because this variable does not represent all aspects related to hand force control and coordination during object manipulation and tasks related to digits dexterity. Also, both JTHFT and 9-HPT, that are used to assess HF, indicate distinct aspects related to the individual's manipulation ability.
\end{abstract}

Keywords: Hand function. Dexterity. Muscle strength dynamometer.

\section{Introdução}

Uma das principais funções da mão (função manual - FM) está relacionada à capacidade do indivíduo em utilizar uma ou ambas as mãos para manipular objetos de diferentes formas, tamanhos, massas e texturas (1). Essa capacidade é indispensável na interação do ser humano com o meio e é crucial para a manutenção de um estilo de vida independente. Para isso, o indivíduo deve ser capaz de controlar e coordenar as forças geradas na interação dedos-objeto $(2,3)$, aplicando uma quantidade de força de preensão que evite o escorregamento do objeto e que não seja excessiva para causar fadiga dos músculos das mãos ou danificar um objeto frágil $(2,4)$. Apesar de a capacidade de controlar a magnitude de força de preensão ser importante, diversos estudos têm avaliado apenas a Força de Preensão Palmar Máxima $\left(\mathrm{FP}_{\text {Max }}\right)$ (5-10) e usado tal variável como indicativo da FM. Assim, pelo fato de os indivíduos terem de controlar a magnitude da força de preensão para realizar tarefas manipulativas e de raramente fazerem uso da $\mathrm{FP}_{\text {Max }}$ para executá-las, podemos questionar a validade dessa variável para avaliar a FM (11).
Além da $\mathrm{FP}_{\mathrm{Max}}$ vários outros testes são utilizados para avaliação da FM, pois não existe um teste considerado como "padrão ouro" para essa avaliação. Dentre os vários tipos de teste, podemos citar aqueles nos quais os indivíduos são solicitados a pegar pinos e inseri-los em buracos pequenos, o mais rápido possível (12-16). Um exemplo desse tipo de teste é o Teste dos Nove Pinos nos Buracos (Rolyan ${ }^{\circledR}$ 9-Hole Peg Test Kit - 9-PnB), que consiste na colocação de nove pinos em nove buracos, seguida da retirada imediata de cada um dos pinos o mais rápido possível $(13,14,17)$. Pelo fato de ambos os testes $\left(\mathrm{FP}_{\text {Max }}\right.$ e 9-PnB) serem indicados como instrumentos de avaliação da FM e por questionarmos a validade da $\mathrm{FP}_{\text {Max }}$, realizamos um estudo que avaliou essas variáveis e constatou uma ausência de relação entre ambas (11). Baseados nesses resultados, concluímos que a $\mathrm{FP}_{\operatorname{Max}}$ não seria um instrumento válido para avaliação da FM em indivíduos jovens sadios. Porém, a ausência de correlação poderia estar associada à escolha de um teste que avalia apenas a destreza dos dedos (i.e., o 9-PnB), e não a função da mão como um todo. 
Um teste bastante utilizado para avaliação da FM global é o Teste de Função Manual de Jebsen e Taylor (TFMJT) (18-20). O TFMJT consiste de tarefas manipulativas semelhantes àquelas realizadas cotidianamente, sendo dividido em sete subtestes: 1) escrita; 2) simulação de uma tarefa de virar cartas; 3) levantamento de objetos pequenos; 4) simulação do uso de colher para a alimentação; 5) empilhar blocos (peças de dama); 6) levantamento de objetos grandes e leves; e 7) levantamento de objetos grandes e pesados. Por se tratarem de tarefas manipulativas mais abrangentes, esse teste parece estar mais relacionado aos vários aspectos que envolvem o funcionamento das mãos e dos membros superiores $(18,19)$. Entretanto, apesar dos inúmeros testes que avaliam a $\mathrm{FP}_{\mathrm{Max}}$ e dos vários outros que avaliam a destreza manual, não há, de forma explícita, nenhum teste que possa ser classificado como "padrão ouro" para avaliação da FM.

Assim, um dos objetivos do estudo foi verificar a relação entre a $\mathrm{FP}_{\mathrm{Max}}$ e o desempenho no TFMJT. Com base no exposto, hipotetizamos que haveria uma relação significativa, porém fraca, entre a $\mathrm{FP}_{\text {Max }}$ e desempenho no TFMJT. Um objetivo adicional deste estudo foi investigar se os desempenhos dos indivíduos no teste 9-PnB e no TFMJT estão relacionados. Essa investigação justifica-se pelo fato de o teste 9 -PnB ser mais simples e mais fácil de ser aplicado do que o TFMJT. E, caso o desempenho no 9-PnB demonstrasse uma forte relação com o desempenho no TFMJT, ele poderia vir a ser uma alternativa à aplicação do TFMJT, tornando-se um indicativo da função manual global.

\section{Método}

\section{Participantes}

Trinta e seis adultos jovens ( 18 homens e $18 \mathrm{mu}-$ lheres) sadios $(24,5 \pm 3$, 8 anos) participaram do estudo, após a assinatura do termo de consentimento livre e esclarecido aprovado pelo Comitê de Ética em Pesquisa, sob no CE/UCS-042/2010, da Universidade Cruzeiro do Sul.

\section{Procedimentos experimentais}

Inicialmente, os participantes preencheram o Inventário de Dominância Lateral de Edimburgo (21), traduzido para a língua portuguesa, para avaliação da dominância manual. Os resultados indicaram 31 indivíduos destros e cinco sinistros. Em seguida, foram aplicados três testes, na seguinte ordem: Teste de Função Manual de Jebsen e Taylor (TFMJT), Teste dos Nove Pinos nos Buracos (9-PnB) e Teste de Força de Preensão Palmar Máxima ( $\mathrm{FP}_{\text {Max }}$ ). Os participantes receberam instruções padronizadas para a execução dos testes, permanecendo sentados confortavelmente para a realização deles. Os participantes realizaram os testes tanto com a mão dominante quanto com a mão não dominante. Finalmente, para evitar efeito de ordem na execução dos testes, metade dos participantes os iniciou com a mão dominante e a outra metade, com a mão não dominante.

O TFMJT foi o primeiro teste aplicado. Apenas seis dos sete subtestes foram aplicados, pois o primeiro deles (isto é, o da escrita) exige conhecimentos da língua inglesa (18). Os participantes sempre receberam a instrução de realizar cada um dos subtestes o mais rápido possível. 0 tempo de execução dos subtestes foi registrado por um cronômetro digital e o desempenho foi avaliado por meio da somatória dos tempos gastos em cada um deles. Como preconizado pelas instruções do TFMJT, os participantes realizaram os subtestes apenas uma vez com cada mão. Nos raríssimos casos em que o participante não conseguiu completar um dos subtestes, em função de algum problema pontual (como queda de objetos do teste no chão), o subteste foi repetido na sequência.

Logo após a execução dos seis subtestes do TFMJT, o teste dos 9-PnB foi aplicado. Esse teste consiste em repetir uma sequência de pegar um pino de 6,4 mm de diâmetro (d) e $32 \mathrm{~mm}$ de comprimento (l), posicionado num recipiente em semicírculo anexo e encaixá-lo num buraco ( $\mathrm{d}=7,1 \mathrm{e} \mathrm{l}=13 \mathrm{~mm})$, repetindo essa sequência por nove vezes, com nove pinos diferentes. Imediatamente após a colocação do nono pino, cada um deles deve ser retirado do buraco e recolocado no recipiente de origem, um por vez $(13,14,17)$. 0 tempo de execução da sequência de tarefas também foi registrado por um cronômetro digital. 0 teste foi repetido três vezes para cada mão, e o menor tempo de execução foi utilizado para análises posteriores. As tentativas para a mão dominante e a não dominante foram realizadas de forma alternada.

Passados aproximadamente dois minutos da realização do teste dos 9-PnB, os participantes realizaram o teste de $\mathrm{FP}_{\mathrm{Max}}$ utilizando um dinamômetro hidráulico de mão $\operatorname{Jamar}^{\circledR}(10,22)$. 0 participante sentado, com o ombro aduzido e rodado neutramente, cotovelo 
fletido a $90^{\circ}$, antebraço pronado a $90^{\circ}$ e punho em posição neutra, foi instruído a segurar o dinamômetro, realizar o máximo de força de preensão possível, apertando com a mão as duas hastes da manopla, durante aproximadamente quatro segundos, e relaxando em seguida. Foram realizadas três tentativas para cada mão, de forma alternada, com intervalo de dois minutos entre elas, evitando qualquer efeito de fadiga $(7,10,23)$. Dentre as três tentativas, o maior valor coletado de força máxima exercida por cada mão foi utilizado como variável dependente $(7,8,10)$.

\section{Análise estatística}

Após verificarmos a normalidade e a homogeneidade de variância dos dados (teste de Shapiro-Wilks), análises de variância (ANOVAs) e testes de correlação de Pearson foram realizadas. Três ANOVAs, tendo como fatores sexo (homem e mulher) e mão (dominante e não dominante), sendo o último fator tratado como medida repetida, foram realizadas para as variáveis somatória dos tempos de execução dos seis subtestes do TFMJT, tempo de execução do 9-PnB e da $\mathrm{FP}_{\text {Max }}$. Análises de correlação de Pearson foram utilizadas para testar a relação entre o desempenho no TFMJT e a $\mathrm{FP}_{\text {Max }}$ e entre o desempenho no TFMJT e no teste dos 9-PnB, para cada uma das combinações sexo-mão (homem-mão dominante, homem-mão não dominante, mulher-mão dominante e mulher-mão não dominante). 0 valor de alfa foi estabelecido em 0,05 .

\section{Resultados}

A Tabela 1 apresenta as médias e os erros-padrão das variáveis analisadas. A Figura 1 apresenta gráficos de dispersão que mostram as relações entre as variáveis dependentes e os respectivos valores de coeficiente de correlação (r) para cada combinação de sexo e mão. Com relação aos efeitos principais de sexo e mão e à interação entre os fatores, as ANOVAs não revelaram diferenças entre mulheres e homens para o desempenho no TFMJT $[F(1,34)=2, p>0,5]$, mas indicaram que as mulheres realizaram o teste dos 9-PnB em menor tempo $[\mathrm{F}(1,34)=13,1, \mathrm{p}<0,005]$, mesmo tendo apresentando menor $\mathrm{FP}_{\text {Max }}[\mathrm{F}(1,34)=52, \mathrm{p}<0,001]$ que os homens. Com relação à mão, as ANOVAs revelaram que, independentemente do sexo, a mão
Tabela 1 - Valores médios (erro padrão) da Força de Preensão Palmar Máxima $\left(\mathrm{FP}_{\mathrm{Max}}\right)$, do desempenho (somatória dos tempos gastos para a execução dos seis subtestes) no Teste de Função Manual de Jebsen e Taylor (TFMJT) e do desempenho (tempo despendido) no Teste dos Nove Pinos nos Buracos (9-PnB), para as mãos dominante (MD) e não dominante (MND), em ambos os sexos

\begin{tabular}{clccc}
\hline & & FP $_{\text {Max }}$ (kgf) & TFMJT (s) & 9-PnB (s) \\
\hline \multirow{2}{*}{ Homens } & MD & $48,72(1,77)^{+,{ }^{\star}}$ & $25,37(0,71)^{\$}$ & $15,47(0,50)^{\$}$ \\
& MND & $45,50(1,84)^{\ddagger, \$}$ & $27,56(0,77)^{\star}$ & $16,39(0,38)^{*}$ \\
\multirow{3}{*}{ Mulheres } & MD & $32,50(1,20)^{+,{ }^{\star}}$ & $24,33(0,41)^{\$}$ & $13,27(0,30)^{\$}$ \\
& MND & $28,00(1,39)^{\ddagger, \$}$ & $26,32(0,57)^{\star}$ & $14,91(0,27)^{*}$ \\
\hline
\end{tabular}

Fonte: Dados da pesquisa.

Legenda: + valor significativamente maior que $\ddagger$, com $p<0,005$;

* valor significativamente maior que $\$$, com $p<0,001$.

dominante obteve melhor desempenho nos três testes realizados, quando comparada à mão não dominante [TFMJT: $F(1,34)=27,14, \mathrm{p}<0,001$; 9-PnB: $F(1,34)=19$, p <0,001; e $\mathrm{FP}_{\operatorname{Max}}$ : $\mathrm{F}(1,34)$ $=24,96, \mathrm{p}<0,001]$. Além disso, as ANOVAs não revelaram qualquer interação entre sexo e mão $(p>0,05)$.

Relação entre a $\mathrm{FP}_{\mathrm{Max}}$ e o desempenho no TFMJT

A relação entre o desempenho no teste de $\mathrm{FP}_{\text {Max }}$ e no TFMJT é apresentada na Figura 1A. As análises de correlação revelaram valores de $r$ significantes para as combinações homem-mão dominante [r (16) $=-0,67, \mathrm{p}<0,005]$, homem-mão não dominante [r (16) $=-0,74, \mathrm{p}<0,001]$ e mulher-mão não dominante $[r(16)=-0,60, p<0,005]$. Esses valores indicam uma correlação negativa de valor moderado entre a $\mathrm{FP}_{\text {Max }}$ e a somatória dos seis subtestes do TFMJT. Em geral, quanto mais forte o indivíduo, menos tempo ele despende para realizar o TFMJT.

\section{Relação entre o desempenho no TFMJT} e no teste dos 9-PnB

A relação entre o desempenho no teste dos 9-PnB e no TFMJT são apresentadas na Figura 1B. As análises 
de correlação revelaram valores significantes de r para as combinações homem-mão não dominante [r (16) $=0,53, \mathrm{p}<0,05]$, e mulher-mão não dominante [r $(16)=0,44, p<0,005]$. Esses valores indicam uma correlação fraca entre os desempenhos nos testes 9-PnB e TFMJT, para ambos os sexos, mas apenas para a mão não dominante.

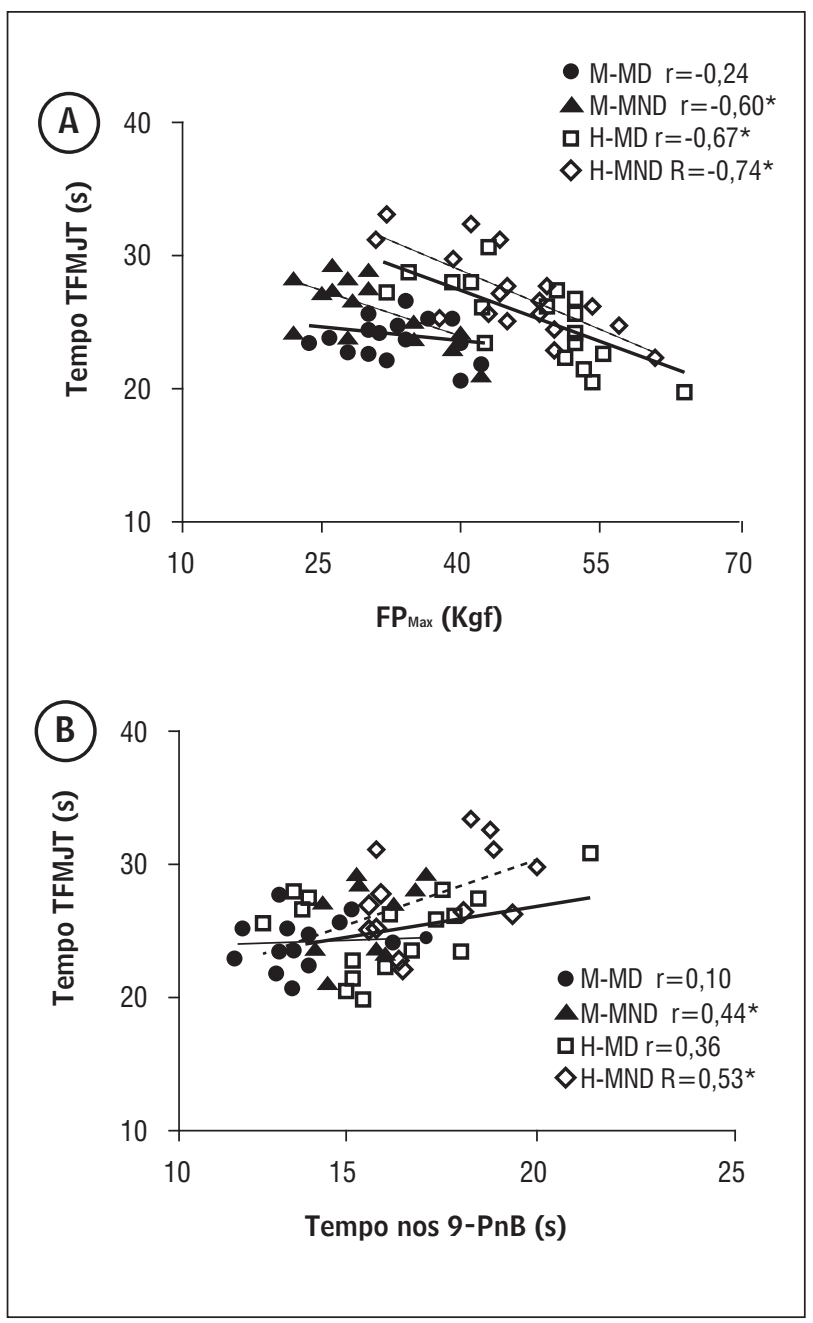

Figura 1 - Gráficos de dispersão apresentam as relações entre as variáveis e os respectivos valores de coeficiente de correlação $(r)$ para as combinações: mulher-mão dominante (M$M D)$, mulher-mão não dominante (M-MND), homem mão dominante (H-MD) e homem-mão não dominante (M-MND).

Fonte: Dados da pesquisa.

Nota: A Figura $1 \mathrm{~A}$ (gráfico de cima) apresenta a relação entre 0 desempenho no TFMJT e a $\mathrm{FP}_{\text {Max }}$. A Figura $1 \mathrm{~B}$ (gráfico de baixo) apresenta a relação entre o desempenho no TFMJT e no teste dos 9-PnB.

\section{Discussão}

Os objetivos do estudo foram examinar a relação entre a $\mathrm{FP}_{\text {Max }}$ e o desempenho no TFMJT e entre os desempenhos no TFMJT e no 9-PnB. Avaliamos homens e mulheres jovens e sadios, que utilizaram suas mãos dominante e não dominante. Primeiramente, testamos os efeitos de sexo e mão, e os resultados revelaram que homens são mais fortes, porém mais lentos na execução do 9-PnB do que as mulheres, e que ambos realizam melhor todas as tarefas com a mão dominante. Baseados nesses achados, correlacionamos as variáveis $\mathrm{FP}_{\text {Max }}$ e desempenho do TFMJT e desempenho no teste dos 9-PnB e no TFMJT, para cada combinação sexo-mão. Em geral, foi observada uma relação inversa entre a $\mathrm{FP}_{\mathrm{Max}}$ e o desempenho no TFMJT, com valores de $r$ menores que 0,74 , e uma correlação positiva, porém fraca $(r<0,52)$, entre o desempenho no TFMJT e no 9-PnB. A seguir, discutiremos esses resultados, iniciando com os efeitos de sexo e mão. Posteriormente, discutiremos as relações entre a $\mathrm{FP}_{\mathrm{Max}}$ e o desempenho no TFMJT e entre o desempenho no TFMJT e no 9-PnB.

\section{Efeitos de sexo e mão \\ para os testes de FM}

Os resultados deste estudo corroboram os achados anteriores que identificaram que homens produzem maior $\mathrm{FP}_{\text {Max }}$ que as mulheres $(5,11,24,25)$. A razão principal seria que, geralmente, os homens possuem maior massa muscular que as mulheres e, consequentemente, são capazes de gerar mais força. Especificamente para o membro superior, essa idéia relaciona-se aos resultados do estudo de Anakwe e colaboradores (24), que verificaram a existência de um relacionamento entre a circunferência do antebraço dos homens (i.e., medida indireta de massa muscular) e a $\mathrm{FP}_{\mathrm{Max}}$. Com relação ao efeito de mão, a maior $\mathrm{FP}_{\mathrm{Max}}$ gerada pela mão dominante poderia ser explicada pelo fato dela ser mais utilizada para a realização de AVDs que exigem produção de força (e.g., segurar e transportar objetos e o uso de ferramentas).

Com relação aos resultados do teste dos 9-PnB, observamos que as mulheres realizaram o teste em menor tempo que os homens. Como não encontramos nenhuma explicação plausível das possíveis causas das diferenças encontradas entre homens e mulheres 
com relação ao desempenho no 9-PnB, poderíamos sugerir que tal diferença seria decorrente da mulher apresentar, em média, a mão e dedos menores que os homens, o que favoreceria um melhor desempenho em tarefas motoras finas. Ainda, poderiamos sugerir que as mulheres foram melhores no teste dos 9-PnB, pois elas, em geral, têm a tendência de executar um maior número de tarefas manipulativas finas e que exigem acurácia no posicionamento final dos movimentos dos dedos (e.g., uso de maquiagem, manuseio de objetos pequenos como brincos). Um dos suportes a essa sugestão é que quando essa exigência de acurácia foi diminuída e movimentos grosseiros foram incluídos (i.e., execução dos subtestes do TFMJT), essa diferença entre sexos desapareceu.

Finalmente, a respeito da diferença entre a mão dominante e a não dominante, se levarmos em consideração a hipótese da especialização hemisférica (2628), que aponta que o membro superior dominante é especializado em controlar e coordenar os movimentos dos segmentos do membro superior ao longo das tarefas realizadas e que o membro não dominante é especializado em alcançar e manter o posicionamento final da mão de forma mais acurada, poderíamos esperar que no teste dos 9-PnB a mão não dominante seria melhor que a dominante. Ainda, considerando essa hipótese, esperávamos que a mão dominante fosse melhor em tarefas que requerem menor exigência da referida acurácia (e.g., TFMJT), o que de fato ocorreu no presente estudo. Entretanto, se analisarmos a tarefa dos 9-PnB detalhadamente, podemos observar que o controle dos movimentos dos segmentos é parte importante da tarefa de colocação dos pinos nos buracos e isso sobreporia a necessidade de acurácia. Isso explicaria o fato da mão dominante ter sido melhor que a não dominante na execução tanto do teste dos 9-PnB $(13,29)$ como do TFMJT $(18-20)$.

\section{Relação entre $\mathrm{FP}_{\operatorname{Max}}$ e o desempenho no TFMJT}

$\mathrm{O}$ fato de raramente aplicarmos $\mathrm{FP}_{\mathrm{Max}}$ em tarefas manipulativas cotidianas gerou um questionamento sobre essa medida ter ou não relação com a FM. Os resultados revelaram uma relação moderada significativa para três das quatro combinações realizadas (i.e., mão não dominante das mulheres e mãos dominante e não dominante dos homens). Esses achados indicam, contrariando o que afirmamos anteriormente (11), que a $\mathrm{FP}_{\mathrm{Max}}$ é uma medida que pode ser utilizada para descrever FM $(5,10,24)$. Porém, essa medida apresenta limitações. Um dos suportes a essa afirmação surge dos resultados encontrados no relacionamento de um dos seis subtestes do TFMJT (a tarefa de pegar objetos pequenos) com o teste de $\mathrm{FP}_{\text {Max }}$. Nós identificamos a mesma ausência de relação entre esse subteste e a $\mathrm{FP}_{\text {Max }}(\mathrm{r}<-0,29, \mathrm{p}>0,05)$ encontrada no relacionamento do teste dos 9-PnB com o de $\mathrm{FP}_{\text {Max }}$ em nosso primeiro estudo (11). Mais uma vez, a FP ${ }_{\text {Max }}$ parece não apresentar qualquer relação com tarefas manipulativas que exigem destreza e controle fino dos movimentos dos dedos, não sendo, portanto, uma medida de força que explique todos os aspectos da FM. De fato, tarefas que exigem maior destreza, como o teste dos 9-PnB, requerem apenas uma aplicação pequena de força de preensão entre os dedos indicador e polegar $(11,30)$.

Possivelmente, muito mais importante do que a capacidade de o indivíduo gerar força máxima é a capacidade de controlar a magnitude da força de preensão ao longo de tarefas manipulativas $(2,3)$. A $\mathrm{FP}_{\mathrm{Max}}$ poderia representar o estado geral do sistema neuromuscular, porém outras variáveis relacionadas à geração de força poderiam descrever melhor o desempenho em testes de FM. Por exemplo, em vez de testes que avaliam níveis máximos da força, poderíamos utilizar variáveis relacionadas à taxa de desenvolvimento de força e outras variáveis neuromusculares relacionadas à produção e ao controle da força em níveis submáximos (31). Essas medidas de força poderiam apresentar relação mais forte com testes funcionais como o TFMJT.

\section{Relação entre os desempenhos no TFMJT e no teste dos 9-PnB}

Procurando entender melhor o quão relacionados estão os testes de destreza manual TFMJT e dos 9-PnB $(14,18)$, aplicamos testes de correlação entre eles para cada combinação de sexo e mão. Nossa hipótese foi a de que iríamos encontrar uma relação de moderada a forte entre ambos e, se fosse esse o caso, poderíamos concluir que apenas a aplicação do teste dos 9-PnB seria necessária para avaliação da FM em indivíduos jovens sadios. No entanto, observamos uma relação fraca entre os desempenhos no TFMJT e no teste dos 9-PnB. Possivelmente, a ausência de uma forte relação entre ambos estaria ligada ao fato de o teste dos 9-PnB limitar-se a avaliar aspectos relacionados com a funcionalidade dos dedos, ao passo que o TFMJT avalia esses e outros aspectos da FM. 
Buscando mais explicações referentes à ausência de relação encontrada entre os dois testes, buscamos novamente correlacionar a tarefa de pegar objetos pequenos do TFMJT com o teste dos 9-PnB. Por serem tarefas semelhantes, que exigem uma preensão de pinça e um maior controle na sua execução, esperávamos que houvesse um relacionamento maior entre as tarefas dos dois referidos testes. Entretanto, não houve correlação significante para nenhuma das combinações $(\mathrm{r}<0,44, \mathrm{p}>0,05)$. A tarefa dos 9-PnB exige que o indivíduo seja rápido e extremamente acurado ao encaixar os pinos nos buracos, ao passo que o subteste do TFMJT exige apenas que o indivíduo seja rápido, sem tanta acurácia da posição final dos objetos depositados na lata. Ademais, a abertura da lata que recebe os objetos é muito maior que o buraco que recebe os pinos (100 e 7,1 mm, respectivamente), exigindo, talvez, que o indivíduo adote estratégias diferentes ao longo da condução dos movimentos do membro superior e dos dedos ao realizar as duas tarefas.

0 resultado encontrado no relacionamento entre o desempenho no TFMJT e no 9-PnB reforça o ponto de que ambos os testes avaliam aspectos diferentes da FM. Assim, com base nos resultados do estudo anterior (11) e do presente estudo, sugerimos que, assim como a $\mathrm{FP}_{\mathrm{Max}^{\prime}}$ o teste dos 9-PnB não seja utilizado como único instrumento de avaliação da FM, a menos que se restrinja a avaliar apenas os aspectos relacionados com o funcionamento e destreza dos dedos, e não da FM global. Testes como o TFMJT parecem ser mais indicados para avaliar a FM global por conter em múltiplas tarefas manipulativas associadas aos movimentos realizados pelas mãos cotidianamente (18-20).

\section{Considerações finais}

A força realizada pelas mãos é importante para a execução de tarefas manipulativas e, consequentemente, da FM. A FP ${ }_{\text {Max }}$ tem sido utilizada como variável importante na avaliação da FM. Baseados no resultado deste estudo, que revelou uma correlação moderada entre $\mathrm{FP}_{\text {Max }}$ e desempenho do TFMJT, podemos concluir que a $\mathrm{FP}_{\mathrm{Max}}$ pode ser utilizada como indicativo da FM, mas seus resultados devem ser interpretados com cautela, haja vista que essa variável não contempla aspectos que envolvem a coordenação e o controle durante a manipulação de objetos. Além disso, com base no resultado que revelou uma correlação fraca entre os desempenhos nos testes 9-PnB e TFMJT, podemos concluir que ambos os testes avaliam aspectos diferentes da função manual e que o teste dos 9-PnB não deve ser utilizado isoladamente para avaliação da FM.

\section{Referências}

1. Jones LA, Lederman SJ. Book human hand function. New York: Oxford University Press; 2006. doi:10.1093/acpro f:oso/9780195173154.001.0001.

2. de Freitas PB, Krishnan V, Jaric S. Force coordination in object manipulation. J Hum Kinet. 2008;20:37-51. doi:10.2478/v10078-008-0016-8.

3. Zatsiorsky VM, Latash ML. Multifinger prehension: an overview. J Mot Behav. 2008;40(5):446-76. doi:10.3200/ JMBR.40.5.446-476.

4. Westling G, Johansson RS. Factors influencing the force control during precision grip. Exp Brain Res. 1984;53(2):277-84. doi:10.1007/BF00238156.

5. Caporrino FA, Faloppa F, Santos JBG, Réssio C, Soares FHC, Nakachima LR, et al. Estudo populacional da força de preensão palmar com dinamômetro Jamar. Rev Bras Ortop. 1998;33(2):150-4.

6. Guerra RS, Amaral TF. Comparison of hand dynamometers in elderly people. J Nutr Health Aging. 2009;13(10):90712. doi:10.1007/s12603-009-0250-3.

7. Haidar SG, Kumar D, Bassi RS, Deshmukh SC. Average versus maximum grip strength: which is more consistent? J Hand Surg Br. 2004;29(1):82-4. doi:10.1016/j. jhsb.2003.09.012.

8. Harkonen R, Piirtomaa M, Alaranta H. Grip strength and hand position of the dynamometer in 204 Finnish adults. J Hand Surg Br. 1993;18(1):129-32. doi:10.1016/0266-7681(93)90212-X.

9. Luna-Heredia E, Martin-Pena G, Ruiz-Galiana J. Handgrip dynamometry in healthy adults. Clin Nutr. 2005;24(2):250-8. doi:10.1016/j.clnu.2004.10.007.

10. Mathiowetz V, Kashman N, Volland G, Weber K, Dowe M, Rogers S. Grip and pinch strength: normative data for adults. Arch Phys Med Rehabil. 1985;66(2):69-74. PMid:3970660.

11. Lima KCA, Santos RQ, de Freitas PB. Relação entre a força máxima e destreza manual em adultos saudáveis: implicações para a avaliação da função manual. Braz J Motor Behav. 2011;6(1):1-6. 
12. Backman C, Cork S, Gibson G, Parsons J. Assessment of hand function: the relationship between pegboard dexterity and applied dexterity. Can J Occup Ther. 1992;59:208-13.

13. Oxford Grice K, Vogel KA, Le V, Mitchell A, Muniz S, Vollmer MA. Adult norms for a commercially available nine hole peg test for finger dexterity. Am J Occup Ther. 2003;57(5):570-3. doi:10.5014/ajot.57.5.570.

14. Mathiowetz V, Weber K, Kashman N, Volland G. Adult norms for the nine hole peg test of finger dexterity. Occup Ther J Res. 1985;5(1):24-38.

15. Mathiowetz V, Rogers SL, Dowe-Keval M, Donahoe L, Rennells C. The Purdue Pegboard: norms for 14- to 19-yearolds. Am J Occup Ther. 1986;40(3):174-9. doi:10.5014/ ajot.40.3.174.

16. Bryden PJ, Roy EA. A new method of administering the grooved pegboard test: performance as a function of handedness and sex. Brain Cognition. 2005;58(3):25868. doi:10.1016/j.bandc.2004.12.004.

17. Poole JL, Burtner PA, Torres TA. Measuring dexterity in children using the nine-hole peg test. J Hand Ther. 2005;18(3):348-51. doi:10.1197/j.jht.2005.04.003.

18. Jebsen RH, Taylor N, Trieschmann RB, Trotter MJ, Howard LA. An objective and standardized test of hand function. Arch Phys Med Rehabil. 1969;50(6):311-9. PMid:5788487.

19. Lynch KB, Bridle MJ. Validity of the jebsen-taylor hand function test in predicting activities of daily living. Occup Ther J Res. 1989;9(5):316-9.

20. Jebsen RH, Griffith ER, Long EW, Fowler R. Function of "normal" hand in stroke patients. Arch Phys Med Rehabil. 1971;52(4):170-4. PMid:5574691.

21. Oldfield RC. The assessment and analysis of handedness: the Edinburgh inventory. Neuropsychologia. 1971;9:97113. doi:10.1016/0028-3932(71)90067-4.

22. Kellor M, Frost J, Silberberg N, Iversen I, Cummings R. Hand strength and dexterity. Am J Occup Ther. 1971;25(2):77-83. PMid:5551515.

23. Fernandes AA, Marins JCB. Teste de força de preensão manual: análise metodológica e dados normativos em atletas. Fisioter Mov. 2011;24(3):567-78. doi:10.1590/ S0103-51502011000300021.
24. Anakwe RE, Huntley JS, McEachan JE. Grip strength and forearm circumference in a healthy population. J Hand Surg Eur Vol. 2007;32(2):203-9. doi:10.1016/j. jhsb.2006.11.003.

25. Schlussel MM, dos Anjos LA, de Vasconcellos MT, Kac G. Reference values of handgrip dynamometry of healthy adults: a population-based study. Clin Nutr. 2008;27(4):601-7. doi:10.1016/j.clnu.2008.04.004.

26. Sainburg RL, Kalakanis D. Differences in control of limb dynamics during dominant and nondominant arm reaching. J Neurophysiol. 2000;83(5):2661-75. PMid:10805666.

27. Sainburg RL. Handedness: differential specializations for control of trajectory and position. Exerc Sport Sci Rev. 2005;33(4):206-13. doi:10.1097/00003677-20051000000010.

28. Wang J, Sainburg RL. The dominant and nondominant arms are specialized for stabilizing different features of task performance. Exp Brain Res. 2007;178(4):565-70. doi:10.1007/s00221-007-0936-x.

29. Koyama T, Domen K, Yokoe M, Sakoda S, Kandori A. Psychometrics of dominant right hand during the 9-hole PEG test: differences between PEG placement and removal. PM\&R. 2011;3(1):40-4.

30. Lee-Valkov PM, Aaron DH, Eladoumikdachi F, Thornby J, Netscher DT. Measuring normal hand dexterity values in normal 3-, 4-, and 5-year-old children and their relationship with grip and pinch strength. J Hand Ther. 2003;16(1):22-8. doi:10.1016/S0894-1130(03)80020-0.

31. Bellumori M, Jaric S, Knight CA. The rate of force development scaling factor (RFD-SF): protocol, reliability, and muscle comparisons. Exp Brain Res. 2011;212(3):35969. doi:10.1007/s00221-011-2735-7.

Recebido: 10/11/2011

Received: 11/10/2011

Aprovado: 07/05/2012

Approved: 05/07/2012 